Original Research

\title{
Filaments and four ordered structures inside a neuron fire a thousand times faster than the membrane: theory and experiment
}

\author{
Pushpendra Singh ${ }^{1,2}$, Pathik Sahoo ${ }^{1}$, Subrata Ghosh $^{3,4}$, Komal Saxena ${ }^{5}$, Jhimli Sarkar Manna ${ }^{1,6}$, Kanad Ray ${ }^{2}$, \\ Soami Daya Krishnananda ${ }^{5}$, Roman R Poznanski ${ }^{7}$, Anirban Bandyopadhyay ${ }^{1, *}$ \\ ${ }^{1}$ International Center for Materials and Nanoarchitectronics (WPI-MANA), Research Center for Advanced Measurement and Characterization (RCAMC) \\ NIMS, 1-2-1 Sengen, Tsukuba, 3050047 Ibaraki, Japan \\ ${ }^{2}$ Amity School of Applied Science, Amity University Rajasthan, Kant Kalwar, NH-11C, Jaipur Delhi Highway, Jaipur, 303007 Rajasthan, India \\ ${ }^{3}$ Chemical Science and Technology Division, CSIR-North East Institute of Science and Technology, NEIST, Jorhat, 785006 Assam, India \\ ${ }^{4}$ Academy of Scientific and Innovative Research (AcSIR), Chaziabad, UP 201002, India \\ ${ }^{5}$ Microwave Physics Laboratory, Department of Physics and Computer Science, Dayalbagh Educational Institute, Dayalbagh, 282005 Agra, Uttar Pradesh, \\ India \\ ${ }^{6}$ Department Electronics and Electrical Communication Engineering, IIT Kharagpur, 721302 West Bengal, India \\ ${ }^{7}$ Integrative Neuroscience Group, Melbourne, VIC 3145, Australia \\ *Correspondence: anirban.bandyo@gmail.com (Anirban Bandyopadhyay)
}

DOI:10.31083/j.jin2004082

This is an open access article under the CC BY 4.0 license (https://creativecommons.org/licenses/by/4.0/).

Submitted: 1 November 2021 Revised: 2 December 2021 Accepted: 16 December 2021 Published: 30 December 2021

The current action potential paradigm considers that all components beneath the neuron membrane are inconsequential. Filamentary communication is less known to the ionic signal transmission; recently, we have proposed that the two are intimately linked through time domains. We modified the atom probe-connected dielectric resonance scanner to operate in two-time domains, milliseconds and microseconds simultaneously for the first time. We resonate the ions for imaging rather than neutralizing them as patch clamps do; resonant transmission images the ion flow $10^{3}$ times faster than the existing methods. We revisited action potential-related events by scanning in and around the axon initial segment (AIS). Four ordered structures in the cytoskeletal filaments exchange energy $\sim 250 \mu$ s before a neuron fires, editing spike-time-gap-key to the brain's cognition. We could stop firing above a threshold or initiate a fire by wirelessly pumping electromagnetic signals. We theoretically built AIS, whose simulated electromagnetic energy exchange matched the experiment. Thus far, the scanner could detect \& link uncorrelated biological events unfolding over $10^{6}$ orders in the time scale simultaneously. Our experimental findings support a new dielectric resonator model of neuron functioning in various time domains, thus suggesting the dynamic anatomy of electrical activity as information-rich.

\section{Keywords}

Neuron imaging; Coaxial electrode; Microstructure; Neuroelectrodynamics; Action potential

\section{Introduction}

The imaging of neural events has been one of the hallmarks of neurophysiology, either by chemically neutralizing the ion channels, doping fluorescent molecules. Thus far, the single ion channels $[1,2]$ are mapped using $10^{3}$ times larger nanoprobe [3] or indirectly seen by the fluorescent doped channel proteins [4-8], wherein the silent ion channels remain undetected. Second, the existing ion-channel scanners [9-11] cannot link a millisecond nerve impulse with the microsecond or nanosecond protein oscillations. Literature is rich in milliseconds and nanoseconds studies but hardly addresses what happens in the microseconds time domain. Historically, a three-order time gap remains unbridged. If bridged, from hours and seconds to femtoseconds, the fulltime scale would be complete with some phenomenon.

The electromagnetic $(\mathrm{em})$ resonance of proteins in the milliseconds and nanosecond time domains has been measured since the 1930s [12-17] as $\mathrm{kHz}$ and $\mathrm{GHz}$ resonance frequencies, respectively. The report of $\mathrm{GHz} \mathrm{em}$ resonance is much older than the millisecond ionic resonance of proteins first observed experimentally in the 1980s-through ionic spike was theoretically proposed in 1907 [18]. In the current neuroscience, milliseconds signal transmissions are measured by neutralizing the ions in the patch-clamp. There is another way to do it, resonantly vibrating the ions and measuring transmitted signal (S21, S12 coefficients) across resonating ions. These two methods are very different. We chose the second protocol, which is not popular among neuroscientists. We use primarily Dielectric resonance microscopy, DRM, for the measurement introduced in $1995[19,20]$. The DRM is wireless, scale-free, looks deep inside a biomaterial; no chemical or physical contact is necessary. However, its potential to image the cell's internal structures interacting in different 
time domains has been speculated but not explored yet. Thus far, just like in conventional biology, DRM has been used as a tool that maps a biomaterial in different time domains separately [21], as if different time domains are not connected.

To correlate different time domains into a singular architecture of time or clocks, identifying a typical biomaterial from the dielectric image is required. Here, we find em resonance as a critical biomarker so that ions, helices, secondary structures of proteins, DNA interacting at different time domains are mapped simply by changing the resonance frequency. Mapping the discrete clocks alone does not provide integrated information architecture of a biological system. A resonance frequency drives a system like a clock, several clocks arranged in a 3D shape build information architecture. For 40 years, time crystal research in biology tried to map biological events as nested clocks [22]. We have advanced it further in neuroscience $[23,24]$. Time crystal is an ordered architecture of clocks model a self-operating biological system. As the clocks run, biological properties are generated.

Thus far, we have succeeded in observing self-similar fractal-like operations in three-time domains, milliseconds, microseconds, and nanoseconds. Those could be achieved by pumping kilohertz, megahertz, and gigahertz signals respectively to the biomaterial and looking into its reflected and transmitted signals surface profile [25]. Recently, we showed that neural network circuits that we see under a microscope are not absolute [23]. The isolated clusters of filaments located in distant neurons could wirelessly link, build circuits neglecting the synaptic pathways. Moreover, using quantum optics with electromagnetic resonance, we showed that at least three ordered structures inside a neuron build electromagnetic vortices, regulating ionic bursts of a neuron [24]. One-to-one correspondence between neuron substructures and the vortex hologram generated by a neuron showed that transformation of electromagnetic to electric potential could happen. However, these observations are fairly abstract to conventional biology that is comfortable to see neuron communications in terms of spikes since 1907 [18] and strongly founded on the finding by Hodgkin and Huxley that filaments inside a neuron are silent. Though contested, a map of substructure firing must be presented for a fair evaluation in competition with the membrane spike.

The filaments dispersed in the cell fluids were known to be silent, i.e., they do not contribute to the potential of the membrane. However, when filaments are packed in neural branches, e.g., axon initial segment, AIS, they might vibrate like synchronized dipoles [23, 24]. Filaments vibrating collectively could generate more than threshold energy to affect membrane potential as a scale-free resonance band connects proteins, filaments, and membranes [25]. However, detailed studies were required to find the geometric structure, whose corner points are resonance frequencies. It is those multiple time domains connected in a geometric shape, which hold invariants of neuron firing. We reported that triplet of triplet resonance band, or a triangle whose corner points hold a triangle inside. If the ionic transmission is blocked between a pair of neurons, they still communicate [26]. The possibility for two distinct communication channels, electrical \& ionic, is often explored in cell [27]. Ionic and non-ionic [28] transmissions together may lead to nonsynaptic firing [29, 30], endogenous firing [31, 32]. For two decades, electromagnetic resonance has been reported on the ion channels and filaments regulating the firing [33-43]. However, simultaneously reading the associated events unfolding at different time scales in a nerve spike is not done yet. Our objective is to invent a tool to characterize both filament conductivity \& ion channel dynamics at a time in a cell, as they differ by $10^{3}$ orders in the time scale.

Though plenty of works on the mechanical resonance of neural or cellular fibers, few reports measure electromagnetic communications through the cell. Cell fluid damps the mechanical resonance since a mechanical vibration requires tension \& physical motion. In contrast, fluid alone cannot dampen most parts of the electromagnetic spectrum since it requires rearranging the dipole, i.e., a pair of charges. Combining milliseconds' membrane response with the dipolar and functional group responses in the nanoseconds-picoseconds time domains means connecting the ionic resonance with the dipolar resonance. However, connecting two diametrically opposite mechanisms is not easy. We need to image the activation and de-activation of ion channels with suitable tools, which did not exist even lately. Since an ion channel [44] opens/closes in 10-20 nanoseconds [44, 45] we need a scanner that rapidly records $(\sim 10 \mathrm{~ns})$ signals simultaneously at multiple time domains. The protein signals would only be recorded at a rate of their natural vibration as the nerve impulse transmits.

Moreover, proteins resonate at different time domains, transmitting ions only in milliseconds. How does this temporal management happen between host protein and guest ions? Our current work on quantum optics to create optical vortices of different angular momentums suggested many periodic structures made of proteins in the neuron. Monochromatic light shined on neurons creates a large condensate of vortices as a hologram. The hologram maps all the clocks; thus, the optical vortex hologram represents a $3 \mathrm{D}$ spatial arrangement of clocks or polyatomic time crystal. Many such clocks inside a protein probably regulate a protein operation in different time domains.

Rapid-frozen, cross-section image of an axon suggests an axon initial segment or AIS is filled with densely packed filaments [46-49]. The popular notion that the filaments float randomly in a fluid in the AIS is incorrect. Some filaments like microtubule are in parallel [46, 47], unidirectional $[50,51]$ with a gap $\sim 50 \mathrm{~nm}[48]$, and nearly continuous [52] in the AIS. Outside AIS, the highly ordered Golgi apparatus isolates the filament-bundles at the axonal or dendritic branch junctions, filamentary continuity breaks. So, each part of the axonal or dendritic branches may appear con- 
tinuous when seen from outside as a membrane. Inside, discrete, isolated filament bundles deliver a different character. However, the Golgi apparatus assists the filaments to remain parallel inside a branch similar to AIS [53]. Each isolated filamentary bundle or a branch could act as a distinct electromagnetic resonator, similar to a tuning fork with multiple distinct resonance frequencies. Unfortunately, the ability of these isolated, independent resonators to absorb electromagnetic signals of particular frequency domains and emit like a separate antenna has not been explored. Our current work is the first attempt to map, theoretically model intricately, and experimentally verify how each branch between two junctions acts as a unique information processing device.

There is another intermediate structure between the filamentary core and the uppermost membrane layer. Just 2$3 \mathrm{~nm}$ below the membrane, actin, beta-spectrin form a periodic rectangular lattice-like structure [54]. It is $\sim 200 \mathrm{~nm}$ cross-sectional rings of beta-spectrin (BS) connected by parallel actin (A) wires (BSA). Together they build a crossbar architecture similar to the one we observe in integrated computer chips. The BSA-ordered structures are found almost everywhere of the neural network, in the glial cells and all dendritic, axonal branches of a neural network $[55,56]$. The BSA is not a singular structure, and it hosts various protein superstructures [57-59]. Several proteins are found to anchor with the grid junctions and specific locations. So, those proteins also become part of the global crossbar architecture. However, the ordering of guest protein molecules disappears if the microtubules in the central region of an axon are dissolved chemically [60]. This is a very important development because it means Hodgkin and Huxley when melting the filaments in the 1950s, did destroy the cylindrical crossbar architecture wrapping the axonal and dendritic branches. The interfacing between the filamentary core and the membrane by highly ordered global crossbar architecture is not an accident. Multiple recent reports suggest that the network of filaments covers $98 \%$ by volume of an axon [46-49]. The filamentary core is bonded to the molecularly thin periodic actin-beta-spectrin hollow cylindrical network [54-60], directly in contact with the membrane above. That is why if the core melts, the crossbar architecture melts spontaneously together.

To this context, our recent work on probing the hippocampal neuron using polarized monochromatic light probed structural symmetry particulars of three distinct regions is important [24]. Each structural symmetry provided a unique ring of light. However, our most important observation was imaging the energy transmission by filaments ignoring the membrane and other architectures across the neural network [23]. The electromagnetic resonance field that emitted and absorbed energy from other non-connected neurons also fed the crossbar architecture. The crossbar architecture has $200 \mathrm{~nm}$ wide rings of proteins covering the cylindrical shape of the protein grid. A pair of such rings brighten up in the dielectric resonance image, which controls the ion chan- nels' opening and closing. Therefore, three layers create a triplet of triplet symmetry of clocks [25], and it's a time crystal that we read using optical photon condensate [24].

Our earlier investigations did not isolate the contribution of each component; joint and superficial accounts were measured. Here, we have built a complete theoretical model of the three structures for the first time, matching theoretically predicted isolated and collective contributions using rigorous experiments. The most important finding reported here is that all prime contributors have threshold resonance frequencies that burst energy. So, we found that the dc potential burst of the membrane is the last or final event in a sequence of ac electromagnetic energy bursts. Membrane firing is not alone.

\section{Materials and methods}

\subsection{Creating an artificial axon initial segment, AIS (HeBHiZ, pronounced as "hevis"), using elementary structures in the theoretical simulator}

Based on the findings noted above, we build a model structure of AIS in the theoretical simulator computer science and technology, CST, particularly to justify that our scanner (Fig. 1a,b) could genuinely measure the signals from filaments \& ion channels together. The core filament region is made of microtubules, and the neurofilaments have three parts. The first element of the dielectric structure is He, derived from the extended range of filaments throughout the axon [52]. The second element is $\mathrm{B}$, the unidirectional polarity of all filaments $[50,51]$. Third, $\mathrm{Hi}$, an equidistant $(\sim 50$ $\mathrm{nm}$ ) lattice-like arrangement of parallel filaments [46-49] in the central core. Then we wrap the three types of dielectrics with the crossbar grid structure as the fourth component. The fourth component is $\mathrm{Z}$, a periodic actin-beta-spectrin lattice coupled to the filamentary core [54-60]. Thus theoretically assembled dielectric material model, HeBHiZ representing an AIS. We cultured neurons and experimentally verified predicted energy exchange between distinct ordered structures of AIS. The tiny filaments vibrate at THz-GHz frequencies as a cascade of resonant oscillations builds the $\mathrm{MHz}$ periodic oscillations to assist the membrane's $\mathrm{kHz}$ ionic spikes. HeBHiZ is the dielectric foundation of ionic bursts.

Now we describe the model construction in CST [61] in detail. The cytoskeleton has three kinds of filaments, microtubule, neurofilaments, and microfilaments (actin). We make all three components using pieces of dielectric materials of various elementary shapes. The elementary functional module's geometric peculiarities are considered when choosing the elementary shapes to build a larger structure. For example, the spirally twisted cylindrical tube is used to emulate the shape of filaments and tube-based spiral loops to replicate the alfa-helices. Tape-like sheets are used to replicate betasheet protein-like structures. Then, by assembling these proteins, we build three kinds of filaments, actin, microtubule, and neurofilaments, of various diameters (5-10 nm). We also build ion channels of the membrane and lipid bilayer with similar dielectric properties. 

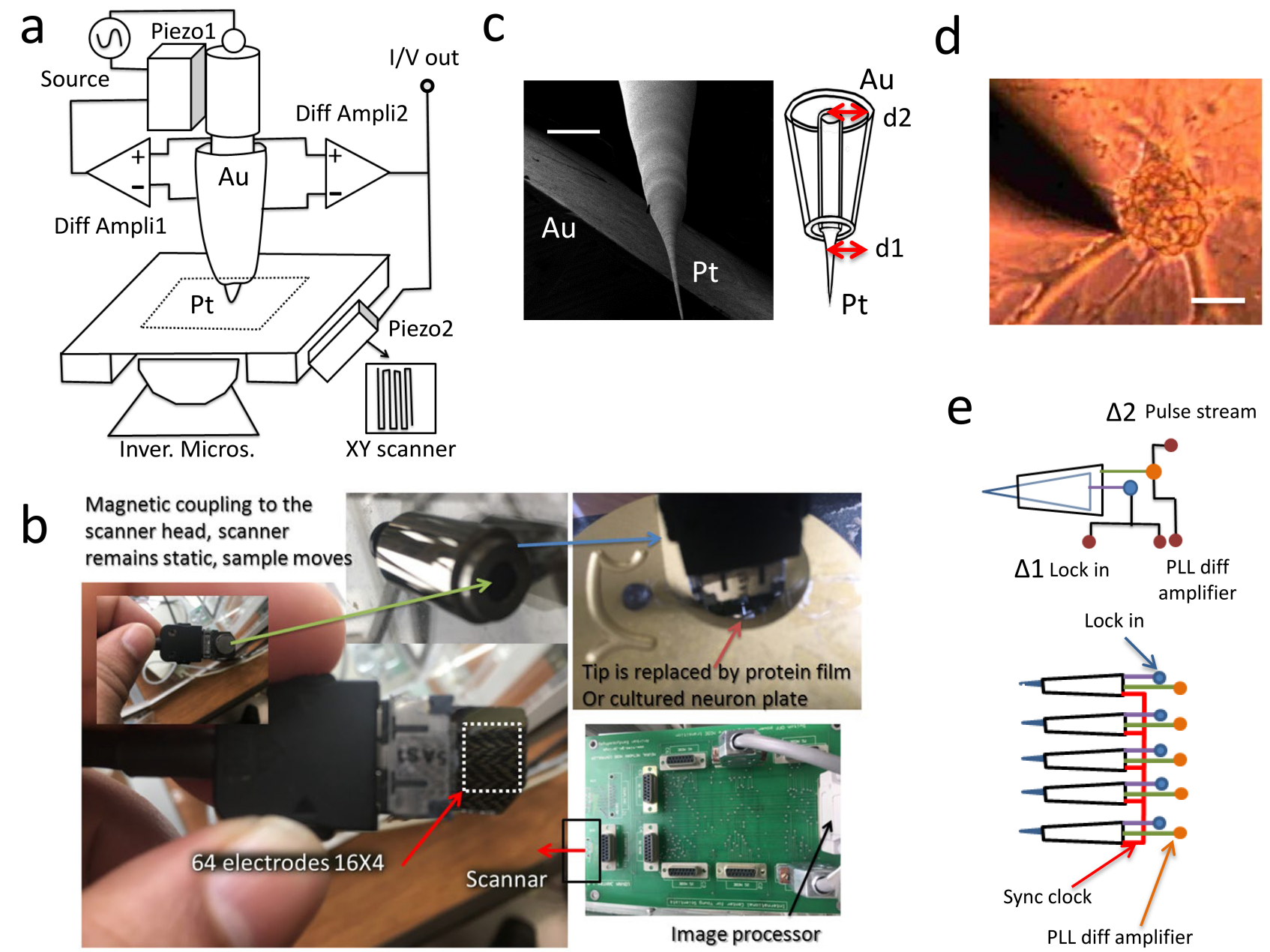

e

$\Delta 2$ Pulse stream
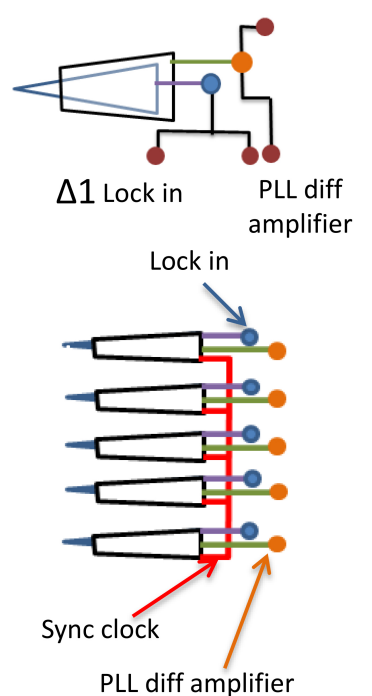

Fig. 1. Basic concepts of SDIM used in the experiment. (a) Double piezo scanner (Piezo1, Piezo2) based Scanning Dielectric Ion Microscope (SDIM) set up for high-resolution non-contact imaging of a neuron membrane \& its internal structure. Diff. Amp., Differential amplifier; Inv Micro, Inverted Microscope. (b) Basic scanning dielectric microscope SDM, which can perform as SDIM, an easy-to-use nano surf STM, was converted to build 64 grid-based simultaneous pixel data capture hardware. (c) He-ion microscopy of coaxial atom probe and its schematic to the right, scale bar $250 \mathrm{~nm}$ (left). Schematic of a coaxial probe. (d) An accurate contact is made to a rat hippocampal neuron using a coaxial probe, scale bar $30 \mu \mathrm{m}$. (e) Coaxial probe measuring circuit connected to one (above) and five coaxial probes. Pulse stream-connected to function generator, PLL diff amplifier-Phase locked loop differential amplifier, Sync periodic oscillation-Synchronized capturing of data sensing \& reading by specialized hardware.

Modeling of electromagnetic resonance of dielectric structures is a common study. Our methodology of creating an artificial theoretical structure is novel and was never attempted before. We do not create a replica of proteins by putting blocks. Rather, we collect the protein structures from the PDB protein database so that when we load them as dielectric helices and tapes in CST, we use an accurate biomaterial structure. Here actin G, ankyrin, beta-spectrin, and tubulin proteins build filaments. Using tubulin, we build a microtubule (25 nm diameter). Using actin $G$, we build actin $F$ filament or microfilament. Finally, to build neurofilaments, five proteins, namely Peripherin, Internexin, Neurofilament protein light (NPL), Neurofilament protein medium (NPM), and Neurofilament protein-heavy (NPH), were used. The crystal structures of the neurofilaments do not reveal an absolute composition of the five proteins. Therefore, we fol- lowed a randomly chosen composition (1:1:3:3:3) to create $10 \mathrm{~nm}$ wide cylinders of various stoichiometry. Neurofilaments have no definite composition \& stoichiometry [62], $\&$ they are not continuous like microtubules. Beta-spectrin size is kept larger than the actin filament since the singlemolecule resonance measurement showed that it absorbs $10^{2}$ times more ac signal energy than the actin filament; we compensated the effect in theory. Therefore, it is an intensive computation, the axon is nearly 10 micrometers wide, and we start from $2 \mathrm{~nm}$-sized protein and intricately build the entire AIS.

In Fig. 2b, blue-colored beta-spectrin hetero dimers align end-to-end to form a complex tetramer that rolls around the central filamentary core like a ring. These beta-spectrin rings are cross-linked by short actin microfilaments extended along the length. This beta-spectrin-actin crossbar-grid binds di- 


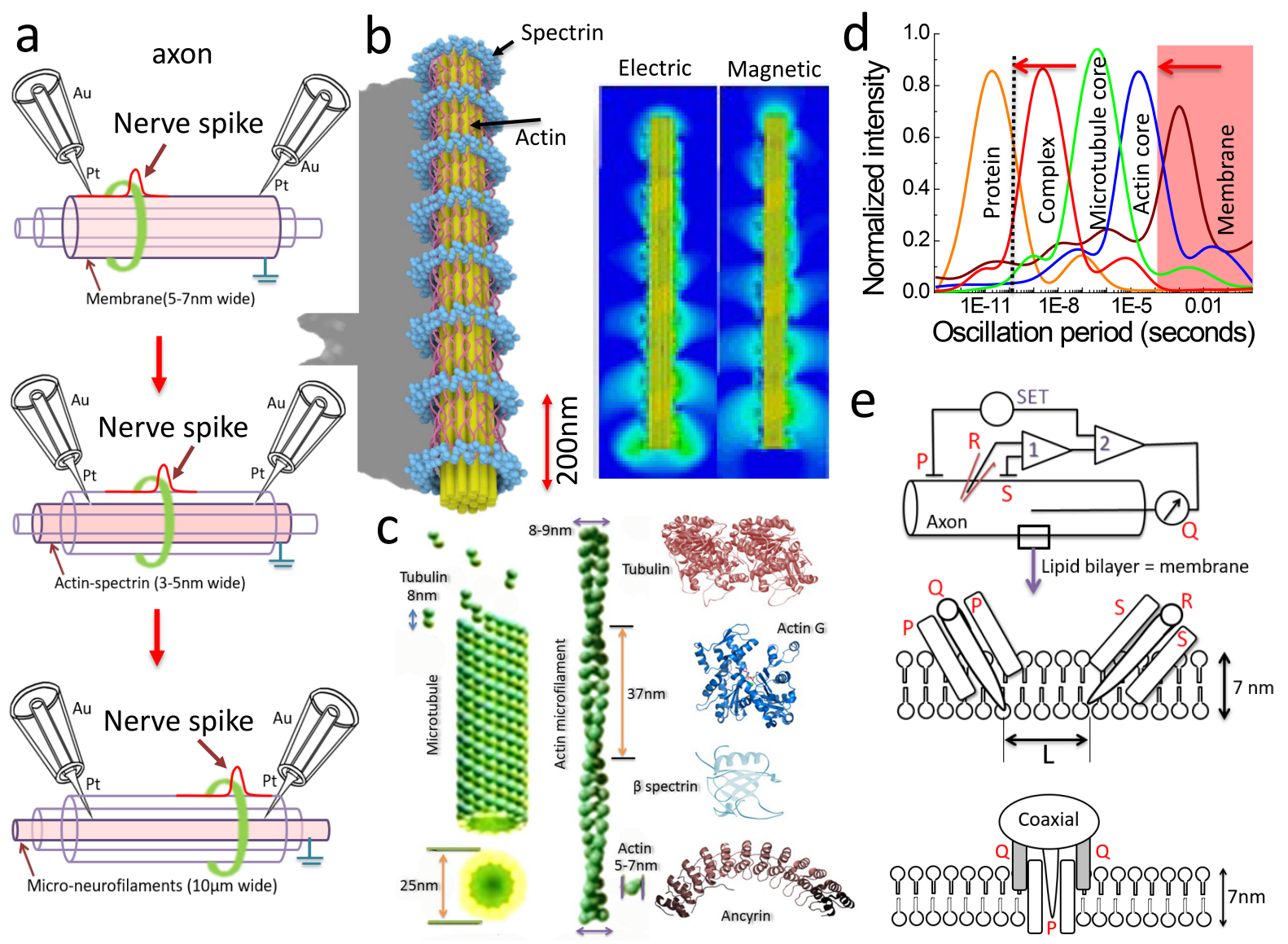

Fig. 2. Measuring natural oscillations deep inside a neuron during firing. (a) Schematic of a pair of coaxial probes entering synchronously first by making contact with the membrane ( $7 \mathrm{~nm}$ wide) (top panel). Then, the coaxial probes are inserted together using a piezo motor to contact the actin-betaspectrin periodic lattice (middle panel). The bottom panel shows the microtubule-neurofilament core. A donut-shaped nerve spike (green) and a linear plot (red) flow left to right as one surfs towards the bottom panel. (b) A schematic of the simulated axon structure ( 8 rings, $\sim 1.6 \mu$ m) built using proteins (left) and its corresponding electric and magnetic field distribution at resonance at $5.5 \mathrm{THz}$. (c) The protein structures tubulin, actin (G), beta spectrin Ankyrin, microtubule, actin filament, tubulin are shown. (d) Coaxial probe measuring the natural oscillation of components, tubulin protein, microtubule $=$ complex, microtubule core = assembly of microtubules \& neurofilaments, actin core = actin-beta spectrin crystal; membrane lipid bilayer. We plot the periodicity of the natural oscillations, averaged over 20 neurons, 437 measurements, $\sim 80$ measurements per region. The shaded region limits a Patch-clamp technique to capture data, and the dotted line is where our probe is most sensitive. (e) The top panel shows old methods using a patch-clamp glass tube or nanotube. The central panel measures a lipid membrane. The bottom panel shows the measurement of a single ion channel. We insert probe directly Pt acquires data, Au holds ground potential (top).

rectly to the plasma membrane; ankyrin protein resides in the grid but binds to the membrane proteins separately. So, the crossbar grid serves as a matrix of thin layer architecture holding many proteins in an ordered arrangement. The central core is made of continuous microtubules and discrete neurofilaments. Continuous microtubules mean that we find a single microtubule from the starting point to the end of AIS. Microtubules do not break in between, but neurofilaments do. The membrane is not part of our axon structure shown in Fig. 2b. We could remove different parts of AIS and simulate preferred frequencies for electromagnetic energy absorption, reflection, and transmission. Then we integrate filaments and proteins into the HeBHiZ structure, as shown in Fig. 2b, accurately following cryo-TEM derived parameters in computer simulation and technology (CST) simulator [61].
The final AIS structure (HeBHiZ) is shown in Fig. 2b. We added two energy supply ports at the two ends of the AIS structure for in silico measurement of reflection and transmission. Only one port is used for simulating the reflection coefficients. In this simulator, we solve Maxwell's equation to derive the reflectance transmittance coefficients (S11 and S21) as a function of frequency. It is called the resonance spectrum.

\subsection{Scanning dielectric ionic microscope, SDIM}

We fused two types of already proven scanning microscopes used for over two decades into a new kind of microscope so that we could measure dipolar and ionic energy exchanges simultaneously. Two microscopes are the scanning ion conductance microscope (SICM, 1989) [9-11] and the scanning dielectric microscope (SDM, 1995) [66]. We call 
it scanning ion dielectric microscope (SICM + SDM = SIDM, see Fig. 1a,b). One major change we made is to use the signal capture probe. We invented the world's smallest patchclamp to measure differential ac and dc conductance using three simultaneous channels at an extremely high signal-tonoise ratio [63]. Since 2016, we have been reporting filamentary firing using these atomic-scale probes with an atomicresolution coaxial electrode $[64,65]$. We must measure both neutralization of ions and dielectric resonance of ions because neutralization of ions has been a technique used since the early days of patch clamp-based action potential measurements. We have been arguing to replace the ion neutralization technique that measures neutralized ion content difference between the Soma's cellular fluid and the buffer solution outside the neuron cell [63-65]. Instead, it is better to resonate the ions, keep them as the active component of the system, thus, do not interfere with the relative ionic balance of the cell and at the same time remotely measure reflectance or transmittance through the ionic clusters in a particular path using resonance spectroscopy. Another advantage of our method is that we could study wide ranges of materials simply by tuning and detuning probe ac signal frequency since each distinct biological material has a distinct resonance frequency peak distribution.

Therefore, in a SIDM, the signal source is a mixture of two frequencies: a millimeter-wave $(\mathrm{GHz})$ to resonate with the measuring protein/molecule and a $\mathrm{kHz}$ wave to resonate with its released ions. When $\mathrm{kHz}$ resonance frequency syncs to read ions released from $0.4 \mathrm{~nm}$ hole of a $2 \mathrm{~nm}$ wide ion channel protein, the second frequency restricts the protein into a single conformer. Thus, the scan resolution increases from $100 \mathrm{~nm}[9,10]$ to $0.2 \mathrm{~nm}$ since the sharp atomic needle makes contact using a piezo motor (Fig. 1c,d). The atomic resolution has already been achieved using scanning dielectric microscopy [67], but not simultaneous recording in multiple time domains. In the SDIM setup (Fig. 1a,b), we use one coaxial probe where a $\mathrm{Pt}(0.1 \mathrm{~nm})$ covered with a dielectric (e.g., glass) is wrapped by a cylindrical $\mathrm{Au} / \mathrm{Cr}(100 \mathrm{~nm})$ layer. The ratio of diameters between starting (d1) and endpoints (d2) of dielectric regulates the probe's sensitivity. Two feedback loops run parallel to guide a probe's piezo motor to make an atomic resolution contact with a single ion channel protein or a $2 \mathrm{D}$ surface scan. It records two signals simultaneously in two-time domains (500 ns and $50 \mathrm{ns)}$ ) at a $10 \mathrm{~ns}$ time gap during approach or scan. So, we visualize ions that vibrate in the milliseconds time domain and dipoles that release ions in the nanoseconds time-domain together using a special setup (Fig. 1e; Fig. 2a).

Two significant technological developments were made, one in electronics and another in the tip. We also changed the ion density measurement protocol since we can truly measure a single ion channel or make contact with the membrane precisely.

Data capture rate: preamplifiers or lock-in amplifiers amplify a very low current $\sim 1 \mathrm{pA}$ but have an integration time of tens of microseconds. So, a firing event is recorded at a gap of $0.2 \mathrm{~ms}$, misses any event happening in-between. If any event regulating a nerve spike begins and ends in microseconds, the existing neurophysiology characterization setups simply cannot detect such events. To achieve a real-time nanoseconds data acquisition, we use a $178 \mathrm{GHz}(5.6 \mathrm{ps}, \sim 0.2 \mathrm{THz})$ function generator connected to a monolithic chip MMIC operating at a $\mathrm{THz}$ speed (MMIC-THz). As a result, the integration of response pulses limits the data capture resolution to $10 \mathrm{~ns}$ (see below for details).

Tip dimension: The nanotubes ( $\sim 20 \mathrm{~nm}$ diameter $)$ are not fit to read a single ion channel. $1 \mu \mathrm{m}(20 \mathrm{~nm})$ wide glass tube reads $\sim 1000(5-10)$ ion channels. Our tip edge is $<0.4 \mathrm{~nm}$, less than the diameter of an ion channel. Still, it requires a mixture of two ac signals for an accurate probing of ions $\&$ the host ion channel protein simultaneously. In dielectric resonance spectroscopy, we do not need to contact the ion channel or dope additional fluorescent molecules as markers of ion release.

Current mode \& voltage mode: When a glass tube clamps with a cell by removing patches from the membrane (Fig. 2e up), the measured current is due to the neutralization of ions. The measured voltage is a potential difference of cell fluid concerning the culture solution connected to the electrical ground. Here, we do not need to patch the probe with the membrane. We can truly measure the membrane. Moreover, our probe is so small that we can truly touch a single ion channel at the junction. Ions resonate with probe frequency, $\mathrm{kHz}$ (Fig. 2e). Therefore, we measure resonance current intensity as ionic current density passing through a single ion channel. Since there is no clamp, we use the term "current mode" and "voltage mode" instead of "currentclamp mode" and "voltage-clamp mode", respectively. Coaxial probes driven by piezo motors are used in the SDIM in the voltage mode to read an ion channel on a $\sim 7 \mathrm{~nm}$ thick membrane. In this mode, SDIM holds the constant current (Fig. 3b,c; Fig. 4a,b,c). The SDIM holds a fixed voltage (Fig. 2d; Fig. 4b-f). By varying the set frequency or editing the search frequency at the SDIM tip, one can contact the invisible \& inaccessible components deep below a membrane.

\subsection{Nanoseconds data acquisition rate}

A set of coaxial atom probes were connected to an active millimeter-wave monolithic integrated circuit (MMICs) based receiver and a transmitter module. Coaxial probes $\mathrm{Au}$ outer shell is fed with $178 \mathrm{GHz}$ pulses, width 5.6 picoseconds, 120 pulsed responses are integrated for each value spending only $0.5 \mathrm{~ns}$; we lose most time afterward. When 120 data was sampled at $220 \mathrm{GHz}$ at the receiver, the integration for signal amplification by $3.4 \mathrm{~dB}$ reduced the resolution to 30 $\mathrm{GHz}$, and noise filtering reduced the sampling resolution to $6 \mathrm{GHz}$. Thus, one ns time resolution in real-time data capture was obtained. A sub-harmonic $178 \mathrm{GHz}$ local oscillator drove the Au tips of both probes, assembled with a broadband quadrature I/Q IF terminals, difference amplifier reduced I/Q 


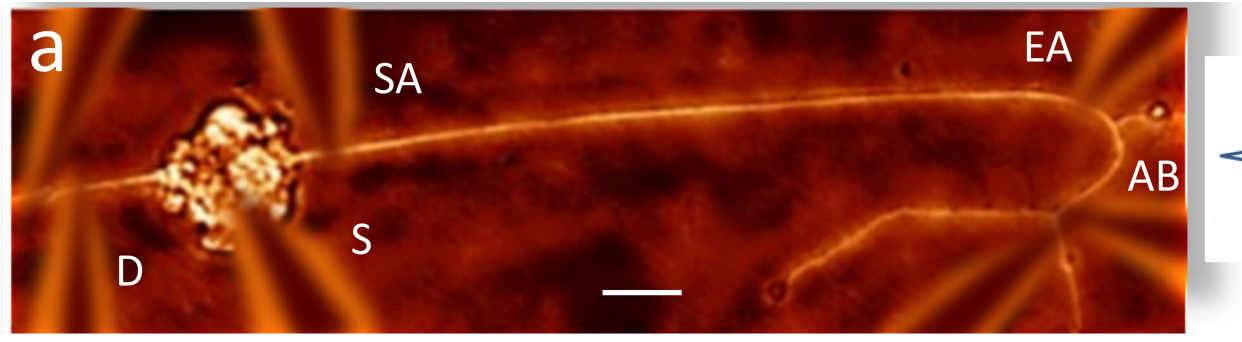

$\Delta 2$ Pulse stream

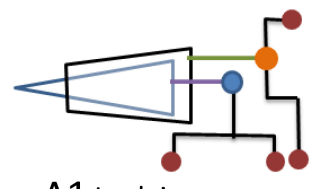

$\Delta 1$ Lock in PLL diff amplifier
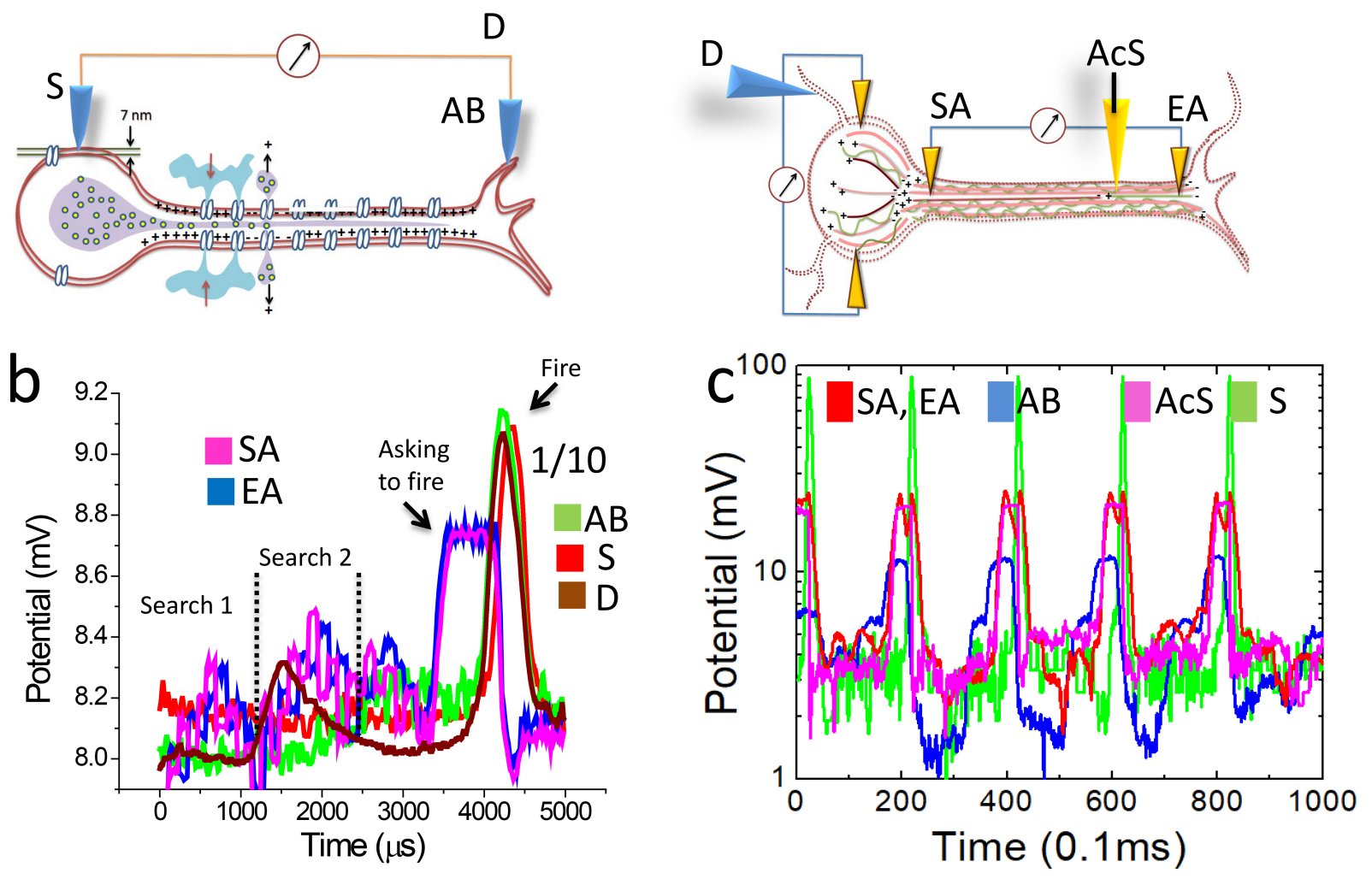

Fig. 3. Measuring natural oscillations deep inside a neuron during firing. (a) Five electrodes are connected to an 8-day old rat hippocampal neuron cultured from embryonic cells, S, Soma; D, Dendrimer; SA, Start of the axon; AIS, Axon Initial Segment; EA, End of axon; AB, Axonal branch. Bottom, a microscope image of a neuron, probes faded as the neuron is focused, scale bar is $50 \mu \mathrm{m}$. S, D, AB make surface contact, EA, SA makes deep axon core contact. (b) Current recording of $5 \mathrm{~ms}$ that includes an ionic firing and current flow at all five probes. The potential ( $\mathrm{mV}$ ) for $\mathrm{S}, \mathrm{D}, \mathrm{AB}$ is reduced by $1 / 10$ to compare all responses in a single time scale [23]. (c) The same plot as panel (b), but for consecutive pulses (total time $100 \mathrm{ms),} \mathrm{the} \mathrm{potential} \mathrm{axis} \mathrm{is} \mathrm{in} \mathrm{log} \mathrm{scale,} \mathrm{AcS,}$ Actin-beta Spectrin lattice.

imbalance below $1.8 \mathrm{~dB}$, increasing output power above 3.5 $\mathrm{dB}$. We read the change in potential at a gap of $10 \mathrm{~ns}$ using a difference amplifier, and we get 100 more data between two consecutive $1 \mathrm{~ms}$ readings.

Millisecond time-domain data were sampled at $40 \mathrm{kHz}$, amplified 300 times using a low noise lock-in amplifier, and a multi-clamp 700B with digidata 1440A (molecular devices A) data acquisition system, filtered using $10 \mathrm{~Hz}$ to the $50 \mathrm{kHz}$ bandpass filter. The peak-to-peak noise level of the device was $(10 \pm 5) \mu \mathrm{V}$. The coaxial probe is similar to the patchclamp, the frequency range is high, and we do not see inverse prepolarization. If it is not an artifact, it will change several basic neuroscience concepts, so we have avoided this issue for further verification by other groups.
2.4 Making precise contact with the desired component deep inside a neuron

The coaxial probe used in this scanner reads an average signal from the $\sim 5-6 \mathrm{~nm}^{2}$ area in the vicinity of the tip. The probe itself affects very few vibrational modes of the measuring system. In the piezo-driven SDM, the current difference between the tip \& the substrate triggers a feedback loop, so the tip lands softly. The membrane vibrates so rapidly that using a circuit similar to Fig. $1 \mathrm{~b}$, we take the tip very close to the membrane surface and wait. Once during vibration, the tip accidentally sticks to the tip edge. The success rate is $66 \%$. We speculate that the resonant coupling between the atomic edge of the tip and the membrane molecule binds strongly. We have described earlier $[64,65]$ that a blind motion of a tip after it penetrates through the membrane can 

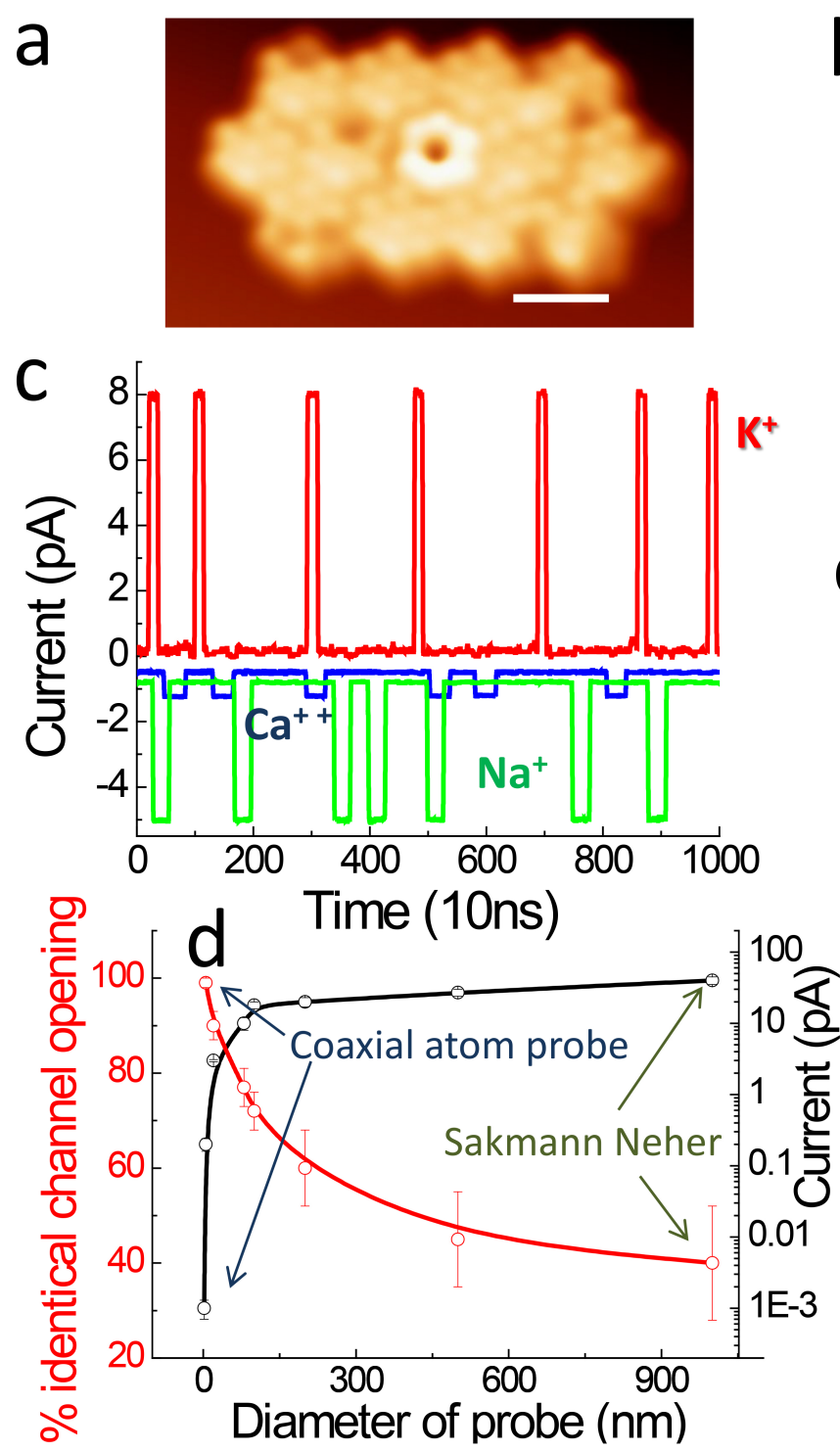
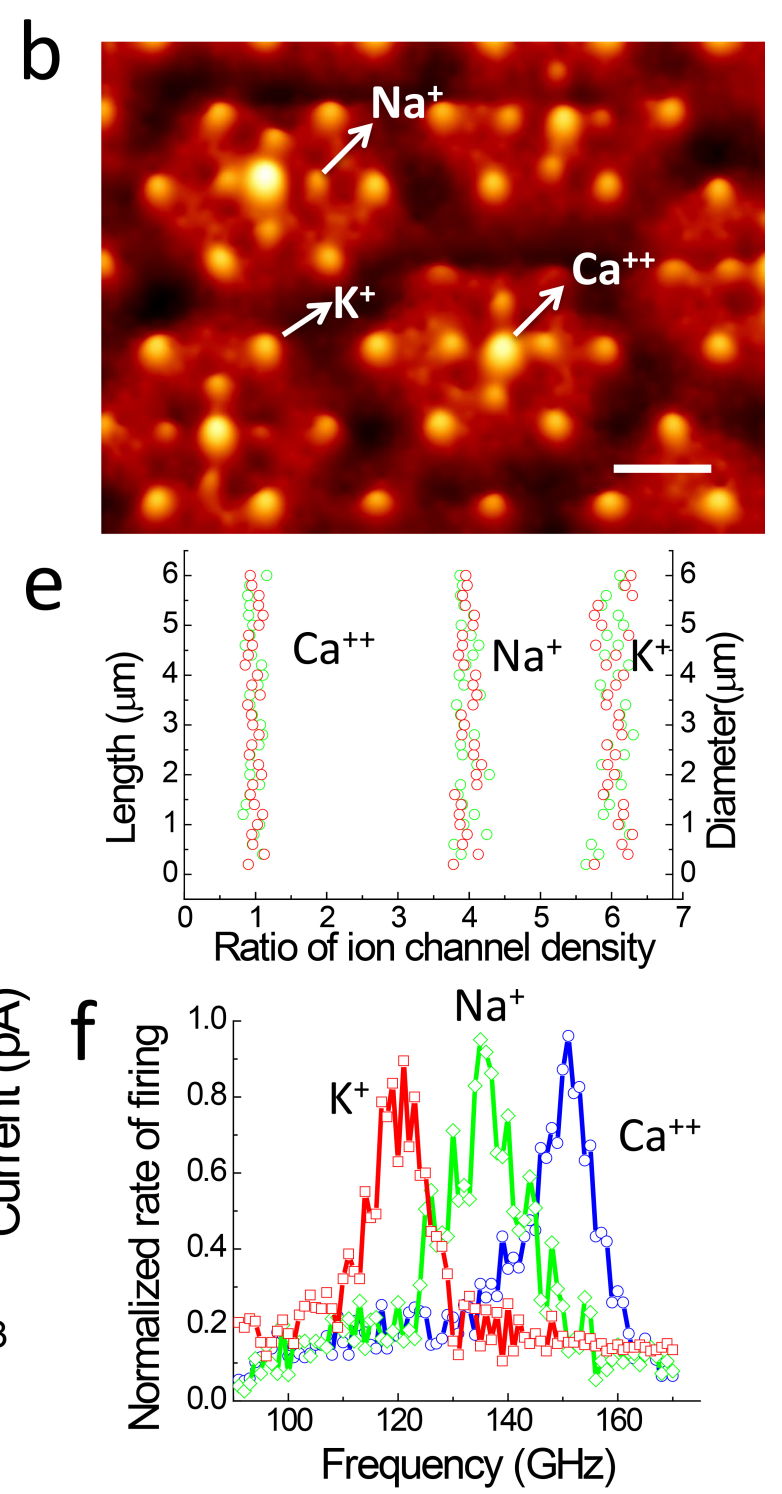

Fig. 4. Imaging \& recording of single ion channel. (a) STM image of an isolated membrane captured by rupturing a live neuron cell, at $2.3 \mathrm{~V}, 6 \mathrm{pA}$. The scale bar is $7 \mathrm{~nm}$. (b) SDIM image of a membrane captured by scanning a live neuron cell at $3.3 \mathrm{~V}, 2 \mathrm{pA}, 130 \mathrm{GHz}$. The scale bar is $16 \mathrm{~nm}$. (c) For Na ${ }^{+}, \mathrm{Ca}++$ and $\mathrm{K}^{+}$ion channels, unitary current amplitude, holding potential $-70 \mathrm{mV}$, coaxial tip Pt had a $5 \mathrm{~nm}$ hole (accidental hole gives the best result) at the edge. Probe fixed at the location shown in panel (b). (d) From $23 \mathrm{Na}^{+}$datasets of panel c, taken by varying probe diameter ( $\left.\mathrm{n}=8\right) 1 \mu \mathrm{m}$ to a single atom ( $0.2 \mathrm{~nm}$ ), how many times a channel remains open for equal time widths, among a random stream of open/close events of ion channels for 8 minutes. Percentage of the opening (black, left) \& current peak as a channel opens (red, right). (e) Sixty branches with different lengths (green) and different diameters (red) were chosen, and shifts in the density relation $\mathrm{Ca}^{++}: \mathrm{Na}^{+}: \mathrm{K}^{+}: 1: 4: 6$ found in panel b are plotted. (f) During natural ion channel Na ${ }^{+}$(green), Ca ${ }^{++}$(blue) and $\mathrm{K}^{+}$(red) open/close event of panel b, an additional ac signal is applied, the rate of the opening is normalized with respect to the $8 \mathrm{MHz}$ maximum rate.

touch the desired structures inside the axon and its branches (8 out of 10 attempts) [63]. However, the probe does not make frequent contact inside Soma. Therein, automated filament search by resonance frequency fails (only 3 out of 10 attempts). Once our probe makes contact, we can confirm by measuring the resonance frequencies (S11 and S21 using VNA, vector network analysis) which structures we have touched. We have already created a database of resonance frequencies of all significant components deep inside a neuron. We match measured values with our database. In this regard, we even opened a neuron cell membrane to find if the resonance frequency-based identification works exactly for studying communication through the architectures inside a neuron. The coaxial probe acts as an optical waveguide \& shields the electrical signal sensor simultaneously. A fusion of dielectric and cavity resonators enables us to set two guiding sensors. The Johnson-Nyquist formula for intrinsic thermal noise is:

$$
\delta V=\sqrt{k_{B} T / C}
$$


where $C=2 \pi \varepsilon L / \ln \left(\frac{b}{a}\right)$, it yields $\delta V=6 \mathrm{mV}$. Our thermal sensing nanowire reduces $\delta V$ apart from the increased resolution by a difference amplifier. Au shield suppresses crosstalk. Glass insulation is enhanced by polymer coating $\left(\sim 10^{12}\right.$ $\Omega)$.

\subsection{The culture of embryogenic hippocampus rat neurons}

We followed the already reported neuron culture with minor changes $[64,65]$. The neuron culture plate was sterilized using $0.01 \%$ poly-l-lysine solution $\left(5 \% \mathrm{CO}_{2}\right)$ overnight at $37^{\circ} \mathrm{C}$. The density of rat hippocampus embryonic cells was kept low $\left(10^{6}\right.$ cells $\left./ \mathrm{mL}\right)$ during cell culture \& post adherence. We observed only those regions where neurons were separated $1 \mathrm{~mm}$ at least. Cell sizes were large, Soma $\sim 1-10 \mu \mathrm{m}$, and axons $\sim 1-100 \mu \mathrm{m}$ long, $250 \mathrm{~nm}-400 \mathrm{~nm}$ wide. We have chosen hippocampal neurons as their propagation failure is a minimum $[68,69](-30 \%)$. We grew neurons from embryogenic cells, the growth saturates 10-15 days, but we used 7-8 days old culture plate for the study. As they change wiring, older neurons do not. We received from Lonza Inc. the embryonic cell suspension of the Rat Brain Hippocampus (Hi); $\geq 0.25 \mathrm{~mL}$. As soon as received, we kept the cells in the Liquid Nitrogen environment $\left(\mathrm{LN}_{2}\right)$. The cell culture protocol was followed as instructed by Lonza Inc, and minor modifications were made [70].

The neuron culture solution is prepared as follows: (1) First, we thaw the Single Quots obtained from Lonza Inc at room temperature $300 \mathrm{~K}$. (2) The basal medium is added to the L-Glutamine and GA vials. (3) We add NSF-1 to the media to reach a final concentration of $2 \%$. Then aliquot the remaining NSF-1 to the desired volume (e.g., $3 \times 1 \mathrm{~mL}$ ) and store the final solution at $-20^{\circ} \mathrm{C}$. (4) Thaw individual NSF-1 aliquots are used to prepare the fresh medium. The survival of the embryonic neurons increases if the osmolality of the PNBM is kept lower $\left(210-240 \mathrm{mOsm} / \mathrm{kg} \mathrm{H}_{2} \mathrm{O}\right)$ than the traditional cell culture media. We supplement PNBM with the PNGM Single Quots, which typically increases the osmolality of a neuron solution by $\sim 10 \mathrm{mOsm} / \mathrm{kg} \mathrm{H} \mathrm{H}_{2} \mathrm{O}$.

We removed a vial of embryonic neuron cells from the liquid Nitrogen chamber $\left(\mathrm{LN}_{2}\right)$ and placed it in a water bath preheated to $37^{\circ} \mathrm{C}$ for 1 minute. We remove the vial, gently transfer $0.25 \mathrm{~mL}$ of neuron cell solution into a $15 \mathrm{~mL}$ centrifuge tube, and immediately add the pre-warmed medium, drop by drop, onto the cells while rotating the tube for $\sim 2$ minutes. Mix the cell suspension by inverting the tube multiple times. We drop the neuron cell suspension to thin HOPG $\& \mathrm{Si} / \mathrm{SiO} 2$ and on a plastic-coated coverslip coated with polyD-lysin film. Incubate the cells dropped on the HOPG \& $\mathrm{Si} / \mathrm{SiO} 2$ substrates for 4 hours at $37{ }^{\circ} \mathrm{C}$ in a $5 \% \mathrm{CO}_{2}$ incubator. We remove the medium from the cells, leaving a thin film of the solution to ensure the cells do not dry out. We constantly add fresh and a pre-warmed medium, replacing the old film. After the initial medium change on day 5 , we replaced $50 \%$ of the growth media every 3 to 4 days. It was warmed at an appropriate amount of medium to $37^{\circ} \mathrm{C}$ in a sterile container. We removed $50 \%$ of the medium from the cell culture. Replaced the culture solution with the warmed, fresh medium and returned the cells to the incubator. Every time after replacing the medium, we incubate the cells at 37 ${ }^{\circ} \mathrm{C}$ with $5 \% \mathrm{CO}_{2}$. We use 5-8 days old rat hippocampus cultured neuron cells (Lonza Inc.) grown on HOPG \& Si/SiO2 and a plastic-coated coverslip. When we grow it on HOPG, we do not need a poly-D-lysin layer to stick the neuron cells.

\section{Results and discussions}

\subsection{Theoretical simulation of axon initial segment, AIS}

We simulated the electromagnetic resonance of an artificial axon (Fig. 2b) by varying its length. Dielectric resonance studies using Maxwell's equations delivered reflectance and transmittance. Anisotropic biomaterials split the electromagnetic signal into asymmetric electric and magnetic field distributions at resonance. Using this property, we provide evidence that if the past experimental findings are true and follow them accurately, the axon structure generates an $\mathrm{MHz}$ periodic oscillation naturally, by theory. At resonance frequencies $3.8 \mathrm{THz}, 5.5 \mathrm{THz}, 7 \mathrm{THz}$, we simulate the spatial distribution of electric and magnetic fields that periodically oscillates $360^{\circ}$ at $5.5 \mathrm{THz}$ through the axonal architecture's surface shown in Fig. 2b,c. The periodic ripples with a microsecond gap depend on the geometry, a function of length, width, and pitch ( $\sim 180 \mathrm{~nm}$ to $220 \mathrm{~nm}$ depending on neurons). There is no explanation, how all ion channels on the perimeter of the circle on the membrane open and close at a time (Fig. 2a). Microsecond periodic oscillations have a spatial periodicity of $200 \mathrm{~nm}$ (Fig. 2b). Spatio-temporal periodic oscillation implies $10^{3}$ times higher frequency signal creates node $\&$ antinodes all along the spiral path inside the axon, precisely in the HeBHiZ structure. Watanabe et al. [71] have shown that transmitted molecules remain in a gap of golden ratio during transmission all along the AIS. In a neuron, faster periodic oscillations integrate into slower periodic oscillations [72]. By removing the neuron membrane, we have measured the resonance of axon structure \& compared it with the membrane $[65,66]$. The consistent resonance band suggests that the dipolar axon architecture HeBHiZ may assist the membrane.

\subsection{Nerve impulse associated signals at different depths inside a neuron}

All neuron data in this work are recorded on the 7-8 days old rat hippocampus neuron cells [73] grown by culturing the embryogenic cells on various substrates described earlier (Fig. 3a) [64, 65]. The schematics below Fig. 3a show two types of contacts between the probe and the material. First, soft physical contact with the membrane surface (left) is shown. Second, the probe is inserted deep below the membrane (right). The potentials at different locations are plotted as a function of time (Fig. 3b,c). The locations are: (i) in the microtubule\& neurofilament bundle in the central part of the axon tube (SA, EA), (ii) on the membrane of Soma (S), (iii) on the membrane of synaptic axon bouton (AB), (iv) actinbeta spectrin cylindrical net, located a few $\mathrm{nm}(3-5 \mathrm{~nm})$ be- 
low the membrane (depicted as AcS) and (v) at the dendritic synaptic bouton (D). Fig. 3c shows four threshold potentials. First, AcS is the filament potential (FP). Second, SA and EA are the action initial segment's potentials (AISP). Third, AB is the synaptic feedback potential (SP). These four potentials set the time of rising, sustain \& fall of an action potential (AP). Here we depict AP as S. The SA \& EA signals in Fig. 3b show two failed attempts to trigger a nerve impulse (depicted as search 1,2 ) before their potentials reach a threshold to hold a flat potential. When SA and EA potentials are flat, energy flow reaches an equilibrium, and that causes the action potential to rise. It is an important observation. Since the technology does not permit us to observe events faster than a millisecond in the membrane-spike view of neuron firing, such signals remained unnoticed. The feedback potential $\mathrm{AB}$ acquires a more organized behavior under the wave train (Fig. 3c) than an isolated discrete pulse (Fig. 3b). Fig. 3c suggests that quantized energy transfer reveals multi-modal communications deep inside a neuron before a neuron fires. Pre-polarization is a fast spike with reverse polarity. It is followed by a nerve spike and is naturally observed in a patchclamp (90 mV) [49] measurement. A reverse polarization is $(\sim 20 \mathrm{mV})$ not observed when a coaxial atom probe reads a nerve impulse. The observation is expected because the measurement mechanism of action potential here is not neutralization of cell fluids but measurement of dielectric property of ions or dipoles. Thus, observation is not limited by fluid flow.

Ion channels of the membrane are connected to a few proteins beneath, which link to the actin-beta spectrin cylindrical net that links the microtubule-neurofilament core. If we calculate the spatial ratio, $95 \%$ of a neural branch is densely packed with microtubule-neurofilament structure, i.e., HeBHiZ. Chemically, Hodgkin-Huxley has shown earlier in their Nobel-prize winning work [74] that neuron fires even if HeBHiZ does not exist. HeBHiZ is not essential for firing. However, our theory and experiment both argue that it edits the time gap between spikes, essential for brain cognition. If the microtubule-neurofilament structure is dissolved, then the membrane attached actin-spectrin periodic lattice-like architecture disappears. Their physical relationship was never measured before. Fig. $2 \mathrm{~d}$ shows the normalized current $(\sim 10$ $\mathrm{pA}$ ) at $100 \mathrm{mV}(\mathrm{RMS})$ ac signal (time $=1 /$ frequency) for tubulin protein, microtubule-neurofilament core, actin-beta spectrin net, and the membrane-measured deep inside a neuron cell. The Gaussian distribution of around 80 measurements suggests that physically isolated neuron components are also temporally isolated. Since many measurements are averaged, we could conclude that five temporal regions exist. We reported the measurement of biomaterial's triplet of triplet resonance bands in these materials earlier [75]. Here, we measure the current response, not the reflected or transmitted pulse train, so it is an average current output of several proteins and complexes. By modifying the probe design, we could extend the operational time limit to record a wide range of elements in the axon. Since in the last 80 years, hundreds of publications reported dielectric resonance of many proteins, we could write a database of frequencies in the control software and detect proteins \& structures if touched during a blind motion through the forest of proteins in an axon. We call it a protein hunt.

Ion channels do not need ATP (ambient temperature and pressure), so even an isolated membrane slice open/close ion channels release ions. We scanned the potential distribution for natural ion emissions from a membrane slice using a Scanning Tunneling Microscope (STM) [23, 24] (Fig. 4a). In SDIM imaging, the lipid molecules of the membrane are not visible, which improves the ion channel mapping (Fig. 4b). Thus far, a patch-clamp always recorded that a single ion channel remains open or close for random durations. For a coaxial probe at tip diameter $<20 \mathrm{~nm}$, an ion channel remains open for a fixed time. Every ion channel (e.g., $\mathrm{Na}^{+}, \mathrm{Ca}^{++}$ and $\mathrm{K}^{+}$) has a characteristic opening period. A SIDM scans the actual composition of ion channels on the membrane, e.g., the density ratio of $\mathrm{Ca}^{++}: \mathrm{Na}^{+}: \mathrm{K}^{+}: 1: 4: 6$. A membrane edits the ratio to modulate a nerve spike (Fig. 4a-e). Each type of ion channel has a distinct frequency band where it opens/closes at the maximum speed (Fig. 4f) [76-78]. In the patch-clamp-based single ion channel measurement, the ionic current is high. As the tip width decreases, the current decreases $10^{3}$ times, i.e., femtoampere $\left(10^{-15} \mathrm{~A}\right)$. Note that $10^{4} \mathrm{ions} / \mathrm{ms}$ is the ion channel conduction rate. It means $1-$ $10 \mathrm{pA}$ to measure the sub-structure conduction. Ideally, if MMIC-THz hardware is faster, one could measure one attoampere (10-18 A) in the future. It means six single charge ions $\left(\mathrm{Na}^{+}, \mathrm{K}^{+}\right)$or three double-charged ions $\left(\mathrm{Ca}^{++}\right)$passing through an ion channel that would be the ultimate and accurate characterization of an ion channel. If filaments regulate the endogenous field, it can trigger ion channels anywhere on the membrane, build multiple threshold potentials [79]. Nonsynaptic mechanisms could control the synchronized firing [29], though controversial, rhythmic nonsynaptic firing events are reported widely even in the absence of synaptic transmission [30]. Consequently, endogenous fields alone could fire [31], i.e., autonomous firing has no pre-initiation, soma potential does not build up from dendritic input [32].

We measured the ion channel density for various lengths and diameters of the axonal branches to confirm the unique ratio of the density of ion channels $\mathrm{Ca}^{++}: \mathrm{Na}^{+}: \mathrm{K}^{+}: 1: 4: 6 \mathrm{ob}-$ served in Fig. 4b. We determine the stoichiometry of ion channels by sensing only the active ones but also in real terms. For a particular class of neurons (polar, bipolar, triangular), neural geometry changes the ion density ratio [80] but not broadly. Geometric ratio $G R=\frac{d_{\text {daughter } 1}^{3 / 2}+d_{\text {daughter } 2}^{3 / 2}}{d_{\text {mother }}^{3 / 2}}$ [81], works in a perfect radial or spherical symmetry. If not symmetric, it fails [82]. As observed in the GR relation above, a particular neuron class holds a strict mathematical relationship, $\mathrm{n}=$ $3 / 2$. However, we studied 27 cases where $n=5 / 3$ ( 8 cases), $n$ $=8 / 5$ ( 19 cases) ratios organized the time editing of the neu- 
ral pulse. Returning to metabolic material transport for long distances $(>1 \mathrm{~cm})$ [83], we speculated that long-distance electromagnetic communication might save a pulse. To verify our hypothesis, we studied the ion channel opening/closing rate as a function of frequency in Fig. 4f, just like Fig. 2d but using wave trains. We repeat that our technology does not allow ultrafast integration at $>0.2 \mathrm{THz}$ ).

Is it possible to regulate an ionic nerve impulse using the resonance frequencies of the components in the filamentary core of an axon that disrupts the three additional signals in Fig. 2b,c? Heating \& cooling of a neuron is the EM effect [36], and heating changes the membrane potential [38]. Infrared $\mathrm{THz}$ electromagnetic signals could edit the nerve impulse as an alternative tool to the ion channels [33-35]. A conduction failure could occur in an ion channel as a function of temperature. Neuron's electromagnetic response is not limited to the infrared region only. A nerve emits $\mathrm{MHz}$ radio waves [40] and is extremely sensitive to microwave [41, 42]. Fig. 5a shows that we apply a sub-threshold pulse to the Soma, not firing. We apply an electromagnetic signal of $22 \mathrm{MHz}$ into the filament bundle of an axon core by bringing a Yagi antenna to its vicinity. The electromagnetic signal is applied in addition to the sub-threshold pulse train. Much to our surprise, the neuron exhibits a continuous above threshold ionic firing under a wave train of sub-threshold pulses.

Bidirectional electromagnetic control of the hypothalamus regulates feeding and metabolism [43]. $\mathrm{Ca}^{2+}$ ion channel proteins could be switched on-off using a mobile phone like wireless devices [42]. Consequently, we tried to shut down an inevitable firing using an ac signal. Our effort was selecting resonance frequencies (S11, S21), with positive \& negative refractive indices of actin-spectrin periodic structure \& microtubule-neurofilament structure (HeBHiZ) from our theoretical study described here. We did another experiment in Fig. 5b, just opposite in concept to that described in Fig. 5a. Fig. 5b shows that one could silence the firing of a particular neuron even when above threshold pulses are applied after mixing an additional set of frequencies $(12 \mathrm{MHz}$ + $35 \mathrm{MHz}+7 \mathrm{GHz}+13 \mathrm{GHz})[23,24,64]$ which disrupts the microtubule-neurofilaments (precisely HeBHiZ) signals. A similar selective suppression was reported earlier using an infrared wave $[50,51]$.

Additional three signals that pass through the filaments are not essential for the firing process, but they regulate the frequency modulation \& the multiplexing ability of a neuron via HeBHiZ. We reproduce the previous reports of electromagnetic tuning of neural processes wirelessly with our new tool. The only difference is that now we can say how the previous reports work in reality. Finally, in Fig. 5c, the coaxial probe detects that a membrane's internal structure HeBHiZ has dipoles that oscillate and sends signals, even if there is no firing. We placed two probes at two locations in the axon core and two on the membrane (Fig. 2a), and by synchronizing the four probes as shown in Fig. 1e, we determined that the natural pulses propagate as shown in Fig. $5 \mathrm{c}$ reaches
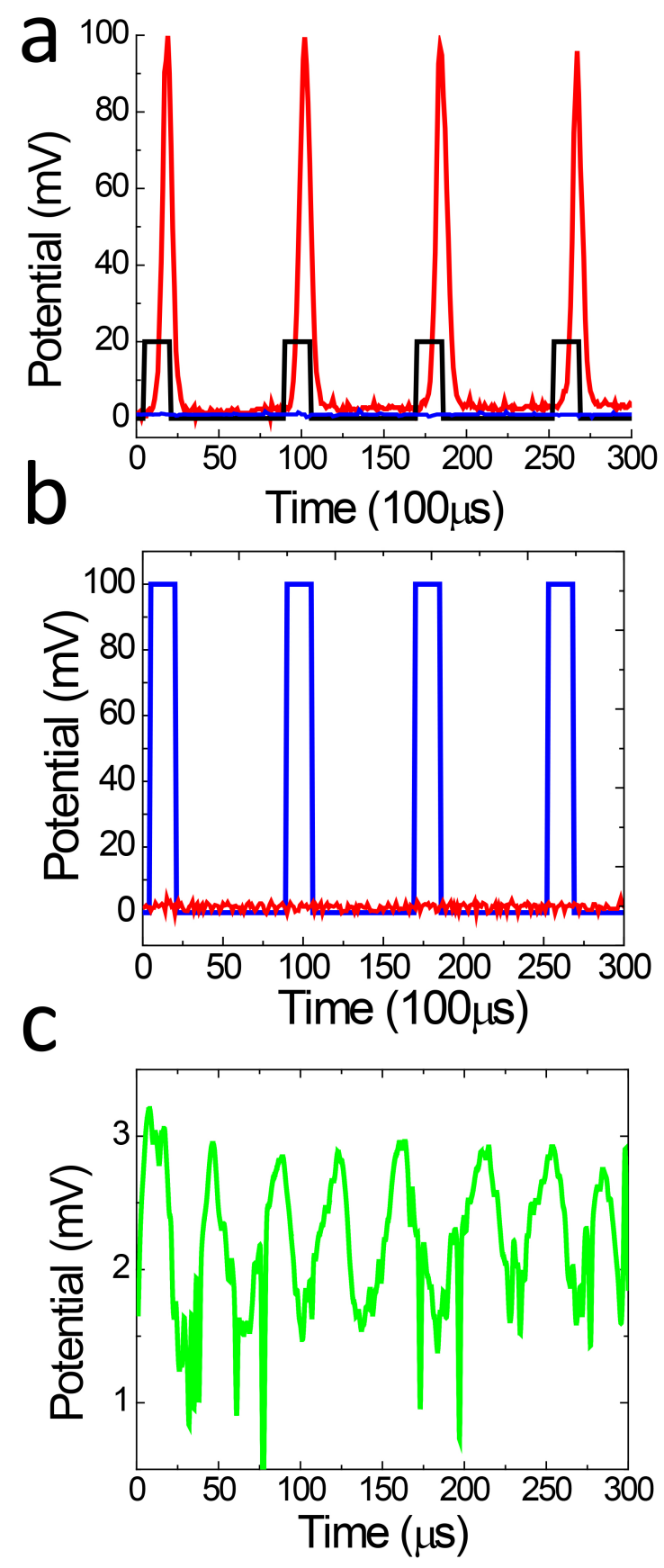

Fig. 5. Electromagnetic editing of a stream of the nerve impulse. (a) 20 $\mathrm{mV}$ stream of sub-threshold pulses applied to the neuron membrane (black). Two output membrane readings are plotted with (red) and without (blue), applying an additional ac frequency. Here, $12 \mathrm{MHz}+35 \mathrm{MHz}+7 \mathrm{GHz}+13$ $\mathrm{GHz}$ is a set of ac frequencies applied to the microtubule-neurofilament core using a coaxial probe inside an axon core. (b) Above threshold $97 \mathrm{mV}$ pulse stream is applied along with a set of ac frequencies $(12 \mathrm{MHz}+35 \mathrm{MHz}+7$ $\mathrm{GHz}+13 \mathrm{GHz}$ ) to the microtubule-neurofilament core (blue). The potential response on the membrane is recorded by contacting the coaxial probe at Soma. (c) $5 \mu \mathrm{m}$ deep inside a coaxial axon probe is inserted, the natural potential fluctuation is recorded when there is no firing.

the destination $10^{3}$ times faster than an ionic wave passing through the membrane. These three findings argue that if an 
SDIM integrates the data faster in the future, it might detect how the filamentary vibrations globally regulate the density of ion channels.

In summary, we triggered a millimeter-wave to fire a neuron even using a sub-threshold pulse where it should not fire by conventional wisdom [76] (Fig. 5a). We could even stop the inevitable firing under an above threshold pulse when mixed with a suitable millimeter-wave [84, 85] (Fig. 5b). A membrane fires even without the filaments inside. The filaments only modulate the spike frequency [75]. That is why even when the neuron does not fire, a natural wave flows through the filaments (Fig. 5c). The whole neuron turns to an integrated vibrating system where distant ion channels, irrespective of their separation, are coupled to signal each other $10^{3}$ times within a span of a single nerve spike via filaments. Thus, a deep neural substructure $[53,64]$ is the third nonsomatic contributor to a nerve spike, in addition to the current axonal computing [82] and gliotransmitters editing the synapse activity. We envision that the improved version of SDIM would reveal many more different signals at various time scales.

\section{Conclusions}

Using a newly developed microscope SDIM, we could image even those ion channels which are not transmitting ions. Our microscope read the dielectric resonance signature by artificially sending signals and reading the reflected and transmitted signals from the materials. Unlike light or infra-red waves, we use the microwave and radio waves to bridge the milliseconds $\left(10^{-3} \mathrm{~s}\right)$ and nanoseconds $\left(10^{-9} \mathrm{~s}\right)$ domains by capturing events that happen in the microsecond $\left(10^{-6} \mathrm{~s}\right)$ time domain in the cell. The SDIM's multi-channel submillisecond signal tracking reveals that at least three simultaneous wave trains flow deep inside a neuron during firing. The HeBHiZ structure was built intricately. For the first time, we have included all significant structural advances in the last two decades into one singular model to understand the time tuning of neurons more precisely. A nerve spike is not limited to the membrane spiking for milliseconds. Its signature is rooted deep below, even in the protein complexes of the microtubule-neurofilament core $\mathrm{HeBHiZ}$, wherein rapid nanosecond pulses regulate the membrane's density of ion channels. The SDIM's recording (10 ns) ability is as fast as a single protein vibrates naturally. Hence, the detection of microsecond pulse streams seems credible. Our finding suggests that different waves driven by different carriers coexist with the ionic spike, still awaiting discovery. Since editing the time gaps between nerve spikes is key to the brain's cognition, the link between $1 \mathrm{~ms}$ and $10 \mathrm{~ns}$ time domains is a primitive yet essential step towards a comprehensive understanding of the neuroelectrodynamic signature of the action potential propagation.

The HH model [86] is part of the dynamics and interaction of electric charges of different temporal scales inside the neuron shaped by molecular interactions, regulating genes, protein expression. These phenomena remodel how electric interactions are performed and implicitly how information is processed and stored inside the neuron. They are required to achieve real-time information processing and bridge between the electrical nature of the brain (including AP generation) and intrinsic information processing at a molecular level. More importantly, the $\mathrm{HH}$ model does not describe the process of physical interaction when information is 'read', 'written' or processed within the neuron. Our series of experiments indicate that signals and signs in the nervous system are information-rich [87]. A single neuron is nested within and above the network of clocks, and geometry made by differential clocks holds the information as a polyatomic time crystal, suggesting that the brain is not a linear Turing tape but rather a fractal tape. The two concepts are orthogonal to each other.

\section{Author contributions}

AB designed the work; SG did the neuron work; PSi, SDK and $\mathrm{KR}$ did the axon theory; KS helped in the experimental set up of dielectric resonance; PSi, AB and PSa wrote the paper; JSM assisted in experiment and data analysis; RRP critically discussed the work.

\section{Ethics approval and consent to participate}

Not applicable.

\section{Acknowledgment}

Thank Dave Sonntag and Martin Timms for our device's independent test \& verification as part of patent US9019685B2.

\section{Funding}

Authors acknowledge the Asian office of Aerospace R\&D (AOARD), a part of the United States Air Force (USAF), for Grant no. FA2386-16-1-0003 (2016-2019) on the electromagnetic resonance-based communication and intelligence of biomaterials.

\section{Conflict of interest}

The authors declare no conflict of interest.

\section{References}

[1] Zhou W, Wang YY, Lim T, Pham T, Jain D, Burke PJ. Detection of single ion channel activity with carbon nanotubes. Scientific Reports. 2015; 5: 9208.

[2] Ide T, Takeuchi Y, Aoki T, Yanagida T. Simultaneous optical and electrical recording of a single ion-channel. The Japanese Journal of Physiology. 2002; 52: 429-434.

[3] Fu T, Duan X, Jiang Z, Dai X, Xie P, Cheng Z, et al. Sub-10-nm intracellular bioelectronic probes from nanowire-nanotube heterostructures. Proceedings of the National Academy of Sciences of the United States of America. 2014; 111: 1259-1264.

[4] Yamamura H, Suzuki Y, Imaizumi Y. New light on ion channel imaging by total internal reflection fluorescence (TIRF) microscopy. Journal of Pharmacological Sciences. 2015; 128 : 1-7.

[5] Bal M, Zhang J, Zaika O, Hernandez CC, Shapiro MS. Homomeric and heteromeric assembly of KCNQ (Kv7) K+ channels assayed by 
total internal reflection fluorescence/fluorescence resonance energy transfer and patch clamp analysis. The Journal of Biological Chemistry. 2008; 283: 30668-30676.

[6] Ulbrich MH, Isacoff EY. Subunit counting in membrane-bound proteins. Nature Methods. 2007; 4: 319-321.

[7] Zheng J, Trudeau MC, Zagotta WN. Rod cyclic nucleotide-gated channels have a stoichiometry of three CNGA1 subunits and one CNGB1 subunit. Neuron. 2003; 36: 891-896.

[8] MacKinnon R, Aldrich RW, Lee AW. Functional stoichiometry of Shaker potassium channel inactivation. Science. 1993; 262: 757-759.

[9] Hansma PK, Drake B, Marti O, Gould SA, Prater CB. The scanning ion-conductance microscope. Science. 1989; 243: 641-643.

[10] Shevchuk AI, Frolenkov GI, Sánchez D, James PS, Freedman $\mathrm{N}, \mathrm{Lab} \mathrm{MJ}$, et al. Imaging proteins in membranes of living cells by high-resolution scanning ion conductance microscopy. Angewandte Chemie. 2006; 45: 2212-2216.

[11] Dutta AK, Korchev YE, Shevchuk AI, Hayashi S, Okada Y, Sabirov RZ. Spatial distribution of maxi-anion channel on cardiomyocytes detected by smart-patch technique. Biophysical Journal. 2008; 94 : 1646-1655.

[12] Elliott MA, Williams JW. The Dielectric Behavior of Solutions of the Protein Zein. Journal of the American Chemical Society. 1939; 61: 718-725.

[13] Pethig R. Dielectric and electronic properties of biological materials (pp. 139). Wiley: New York. 1979.

[14] Vollmer F, Braun D, Libchaber A, Khoshsima M, Teraoka I, Arnold S. Protein detection by optical shift of a resonant microcavity. Applied Physics Letters. 2002; 80: 4057-4059.

[15] Schirò G, Cupane A, Vitrano E, Bruni F. Dielectric Relaxations in Confined Hydrated Myoglobin. The Journal of Physical Chemistry B. 2009; 113: 9606-9613.

[16] Kim Y, Yoon S, Park S, Lim D, Jung H, Kim Y. A simple and direct biomolecule detection scheme based on a microwave resonator. Sensors and Actuators B: Chemical. 2008; 130: 823-828.

[17] Verma R, Daya KS. Rapid Detection of pM Concentration of Insulin Using Microwave Whispering Gallery Mode. IEEE Sensors Journal. 2017; 17: 2758-2765.

[18] Lapicque L. Recherches quantitatives sur 1'excitation electrique des nerfs traitee comme une polarization. Journal of Plant Physiology \& Pathology. 1907; 9: 620- 635.

[19] Asami K. The scanning dielectric microscope. Measurement Science and Technology. 1994; 5: 589-592.

[20] Asami K. Dielectric imaging of biological cells. Colloid \& Polymer Science. 1995; 273: 1095-1097.

[21] Asami K. Characterization of biological cells by dielectric spectroscopy. Journal of Non-Crystalline Solids. 2002; 305: 268-277.

[22] Winfree A. Biological Rhythm Research: The Geometry of Biological Time. Springer: New York. 1977, 2001.

[23] Singh P, Saxena K, Sahoo P, Ghosh S, Bandyopadhyay A. Electrophysiology using coaxial atom probe array: Live imaging reveals hidden circuits of a hippocampal neural network. Journal of Neurophysiology. 2021; 125: 2107-2116.

[24] Singh P, Sahoo P, Saxena K, Manna JS, Ray K, Ghosh S, et al. Cytoskeletal Filaments Deep Inside a Neuron Are Not Silent: They Regulate the Precise Timing of Nerve Spikes Using a Pair of Vortices. Symmetry. 2021; 13: 821.

[25] Saxena K, Singh P, Sahoo P, Sahu S, Ghosh S, Ray K, et al. Fractal, Scale Free Electromagnetic Resonance of a Single Brain Extracted Microtubule Nanowire, a Single Tubulin Protein and a Single Neuron. Fractal and Fractional. 2020; 4: 11.

[26] Katz B, Schmitt OH. Electric Interaction Between Two Adjacent Nerve Fibers. Journal of Physiology. 1940; 97: 471-488.

[27] Ramón F, Moore JW. Ephaptic transmission in squid giant axons. The American Journal of Physiology. 1978; 234: C162-C169.

[28] Jefferys JG. Nonsynaptic modulation of neuronal activity in the brain: electric currents and extracellular ions. Physiological Reviews. 1995; 75: 689-723.

[29] Dudek FE, Yasumura T, Rash JE. 'Non-synaptic' mechanisms in seizures and epileptogenesis. Cell Biology International. 1998; 22: 793-805.

[30] Ren J, Momose-Sato Y, Sato K, Greer JJ. Rhythmic neuronal discharge in the medulla and spinal cord of fetal rats in the absence of synaptic transmission. Journal of Neurophysiology. 2006; 95: 527-534.

[31] Qiu C, Shivacharan RS, Zhang M, Durand DM. Can Neural Activity Propagate by Endogenous Electrical Field? The Journal of Neuroscience. 2016; 35: 15800-15811.

[32] Atherton JF, Wokosin DL, Ramanathan S, Bevan MD. Autonomous initiation and propagation of action potentials in neurons of the subthalamic nucleus. The Journal of Physiology. 2009; 586: 5679-5700.

[33] Westerfield M, Joyner RW, Moore JW. Temperature-sensitive conduction failure at axon branch points. Journal of Neurophysiology. 1978; 41: 1-8.

[34] Duke AR, Jenkins MW, Lu H, McManus JM, Chiel HJ, Jansen ED. Transient and selective suppression of neural activity with infrared light. Scientific Reports. 2014; 3: 2600.

[35] Yoo S, Hong S, Choi Y, Park J, Nam Y. Photothermal inhibition of neural activity with near-infrared-sensitive nanotransducers. ACS Nano. 2015; 8: 8040-8049.

[36] Abbott BC, Hill AV, Howarth JV. The positive and negative heat associated with a nerve impulse. Proceedings of the Royal Society B. $1958 ; 148: 149-187$.

[37] Buzatu S. The temperature-induced changes in membrane potential. Rivista Di Biologia. 2010; 102: 199-217.

[38] Howarth JV, Keynes RD, Ritchie JM, von Muralt A. The heat production associated with the passage of a single impulse in pike olfactory nerve fibres. The Journal of Physiology. 1975; 249: 349368.

[39] Gittes F, Mickey B, Nettleton J, Howard J. Flexural rigidity of microtubules and actin filaments measured from thermal fluctuations in shape. The Journal of Cell Biology. 1993; 120: 923-934.

[40] Fraser A, Frey AH. Electromagnetic emission at micron wavelengths from active nerves. Biophysical Journal. 1969; 8: 731-734.

[41] Partsvania B, Sulaberidze T, Shoshiashvili L. Effect of high SARs produced by cell phone like radiofrequency fields on mollusk single neuron. Electromagnetic Biology and Medicine. 2014; 32: 4858.

[42] Maskey D, Kim M, Aryal B, Pradhan J, Choi I, Park K, et al. Effect of $835 \mathrm{MHz}$ radiofrequency radiation exposure on calcium binding proteins in the hippocampus of the mouse brain. Brain Research. 2010; 1313: 232-241.

[43] Stanley SA, Kelly L, Latcha KN, Schmidt SF, Yu X, Nectow AR, et al. Bidirectional electromagnetic control of the hypothalamus regulates feeding and metabolism. Nature. 2016; 531: 647-650.

[44] Harms G, Orr G, Lu HP. Probing ion channel conformational dynamics using simultaneous single-molecule ultrafast spectroscopy and patch-clamp electric recording. Applied Physics Letters. 2004; 84: 1792-1794.

[45] Tahara S, Takeuchi S, Abe-Yoshizumi R, Inoue K, Ohtani H, Kandori $\mathrm{H}$, et al. Ultrafast photoreaction dynamics of a light-driven sodium-ion-pumping retinal protein from Krokinobacter eikastus revealed by femtosecond time-resolved absorption spectroscopy. The Journal of Physical Chemistry Letters. 2015; 6: 4481-4486.

[46] Hirokawa N. From electron microscopy to molecular cell biology, molecular genetics and structural biology: intracellular transport and kinesin superfamily proteins, KIFs: genes, structure, dynamics and functions. Journal of Electron Microscopy. 2011; 60: S63S92.

[47] Hirokawa N, Pfister KK, Yorifuji H, Wagner MC, Brady ST, Bloom GS. Submolecular domains of bovine brain kinesin identified by electron microscopy and monoclonal antibody decoration. Cell. 1989; 56: 867-878.

[48] Hirokawa N, Shiomura Y, Okabe S. Tau proteins: the molecular structure and mode of binding on microtubules. The Journal of Cell Biology. 1988; 107: 1449-1459.

[49] Chen J, Kanai Y, Cowan NJ, Hirokawa N. Projection domains of 
MAP2 and tau determine spacings between microtubules in dendrites and axons. Nature. 1993; 360: 674-677.

[50] Heidemann SR, Landers JM, Hamborg MA. Polarity orientation of axonal microtubules. Journal of Cell Biology. 1982; 91: 661-665.

[51] Heidemann SR, Hamborg MA, Thomas SJ, Song B, Lindley S, Chu D. Spatial organization of axonal microtubules. Journal of Cell Biology. 1984; 99: 1289-1295.

[52] Baas PW, Deitch JS, Black MM, Banker GA. Polarity orientation of microtubules in hippocampal neurons: uniformity in the axon and nonuniformity in the dendrite. Proceedings of the National Academy of Sciences of the United States of America. 1988; 85 8335-8339.

[53] Xu K, Zhong G, Zhuang X. Actin, spectrin, and associated proteins form a periodic cytoskeletal structure in axons. Science. 2013; 339: 452-456.

[54] He J, Zhou R, Wu Z, Carrasco MA, Kurshan PT, Farley JE, et al. Prevalent presence of periodic actin-spectrin-based membrane skeleton in a broad range of neuronal cell types and animal species. Proceedings of the National Academy of Sciences of the United States of America. 2016; 113: 6029-6034.

[55] D’Este E, Kamin D, Velte C, Göttfert F, Simons M, Hell SW. Subcortical cytoskeleton periodicity throughout the nervous system. Scientific Reports. 2017; 6: 22741

[56] Gervasi MG, Xu X, Carbajal-Gonzalez B, Buffone MG, Visconti $\mathrm{PE}$, Krapf D. The actin cytoskeleton of the mouse sperm flagellum is organized in a helical structure. Journal of Cell Science. 2019; 131: jcs215897.

[57] D’Este E, Kamin D, Göttfert F, El-Hady A, Hell SW. STED nanoscopy reveals the ubiquity of subcortical cytoskeleton periodicity in living neurons. Cell Reports. 2015; 10: 1246-1251.

[58] Lukinavičius G, Reymond L, D’Este E, Masharina A, Göttfert F, Ta $\mathrm{H}$, et al. Fluorogenic probes for live-cell imaging of the cytoskeleton. Nature Methods. 2014; 11: 731-733.

[59] Ganguly A, Tang Y, Wang L, Ladt K, Loi J, Dargent B, et al. A dynamic formin-dependent deep F-actin network in axons. The Journal of Cell Biology. 2016; 210: 401-417.

[60] Zhong G, He J, Zhou R, Lorenzo D, Babcock HP, Bennett V, et al. Developmental mechanism of the periodic membrane skeleton in axons. ELife. 2015; 3: e04581.

[61] CST GmbH. CST MICROWAVE STUDIO ${ }^{\circledR} 2006$ - Rectangular Waveguide Tutorial. 2006. Available at: https://perso.telecom-p aristech.fr/begaud/intra/MWS_Tutorials.pdf (Accessed: 18 December 2021)

[62] Nixon RA, Shea TB. Dynamics of neuronal intermediate filaments: a developmental perspective. Cell Motility and the Cytoskeleton. 1992; 22: 81-91.

[63] Singh P, Ghosh S, Sahoo P, Ray K, Fujita D, Bandyopadhyay A. Reducing the dimension of a patch-clamp to the smallest physical limit using a coaxial atom probe. Progress in Electromagnetics Research B. 2020; 89: 29-44.

[64] Ghosh S, Sahu S, Agrawal L, Shiga T, Bandyopadhyay A. Inventing a co-axial atomic resolution patch clamp to study a single resonating protein complex and ultra-low power communication deep inside a living neuron cell. Journal of Integrative Neuroscience. 2017; 15: 403-433.

[65] Agrawal L, Sahu S, Ghosh S, Shiga T, Fujita D, Bandyopadhyay A. Inventing atomic resolution scanning dielectric microscopy to see a single protein complex operation live at resonance in a neuron without touching or adulterating the cell. Journal of Integrative Neuroscience. 2016; 15: 435-462.
[66] Cho Y, Kirihara A, Saeki T. Scanning nonlinear dielectric microscope. Review of Scientific Instruments. 1996; 67: 2297-2303.

[67] Cho Y. Scanning nonlinear dielectric microscopy with super-high resolution, Japanese Journal of Applied Physics. 2007; 46: 7B.

[68] Raastad M, Shepherd GMG. Single-axon action potentials in the rat hippocampal cortex. The Journal of Physiology. 2004; 548: 745-752.

[69] Mackenzie PJ, Umemiya M, Murphy TH. Ca2+ imaging of CNS axons in culture indicate reliable coupling between single axon potentials and distal functional release sites. Neuron. 1996; 16: 783795.

[70] Kaech S, Banker G. Culturing hippocampal neurons. Nature Protocols. 2007; 1: 2406-2415.

[71] Watanabe K, Al-Bassam S, Miyazaki Y, Wandless TJ, Webster P, Arnold DB. Networks of polarized actin filament in the axon initial segment provides a mechanism for sorting axonal and dendritic proteins. Cell Reports. 2012; 2: 1546-1553.

[72] Connor JA. Slow repetitive activity from conductance changes in neuron. Federation Proceedings. 1978; 37: 2139-2145.

[73] Spencer WA, Kandel ER. Electrophysiology of hippocampal neurons. Journal of Neurophysiology. 1961; 24: 272-285

[74] Hodgkin AL, Huxley AF, Katz B. Measurement of current-voltage relations in the membrane of the giant axon of Loligo. The Journal of Physiology. 2004; 116: 424-448.

[75] Bandyopadhyay A. Nanobrain: The making of an artificial brain from a time crystal (pp. 1-336). Imprint CRC Press Inc.: Bosa Roca, United States. 2020.

[76] Doyle DA, Morais Cabral J, Pfuetzner RA, Kuo A, Gulbis JM, Cohen SL, et al. The structure of the potassium channel: molecular basis of $\mathrm{K}+$ conduction and selectivity. Science. 1998; 280: 69-77.

[77] Sonnleitner A, Isacoff E. Single ion channel imaging. Methods in Enzymology. 2003; 361: 304-319.

[78] Demuro A, Parker I. Optical patch-clamping": single-channel recording by imaging $\mathrm{Ca} 2+$ flux through individual muscle acetylcholine receptor channels. Journal of General Physiology. 2005; 126: 179-192.

[79] Sardi S, Vardi R, Sheinin A, Goldental A, Kanter I. New Types of Experiments Reveal that a Neuron Functions as Multiple Independent Threshold Units. Scientific Reports. 2017; 7: 18036.

[80] Debanne D. Information processing in the axon. Nature Reviews. Neuroscience. 2004; 5: 304-316.

[81] Rall W. Branching dendritic trees and motoneuron membrane resistivity. Experimental Neurology. 1998; 1: 491-527.

[82] Rall W, Shepherd GM. Theoretical reconstruction of field potentials and dendrodendritic synaptic interactions in olfactory bulb. Journal of Neurophysiology. 1969; 31: 884-915.

[83] Ofer N, Shefi O. Axonal geometry as a tool for modulating firing patterns. Applied Mathematical Modelling. 2016; 40: 3175-3184.

[84] Wu J, Xu Y, Ma J. Lévy noise improves the electrical activity in a neuron under electromagnetic radiation. PLoS ONE. 2017; 12: e0174330.

[85] Yoo S, Hong S, Choi Y, Park JH, Nam Y. Photothermal inhibition of neural activity with near-infrared-sensitive nanotransducers. ACS Nano. 2014; 8: 8040-8049.

[86] Hodgkin AL, Huxely AF. A quantitative description of membrane current and its applications to conduction and excitation in nerve. Journal of Physiology. 1952; 117: 500-544.

[87] Bullock TH. Signals and signs in the nervous system: the dynamic anatomy of electrical activity is probably information-rich. Proceedings of the National Academy of Sciences of the United States of America. 1997; 94: 1-6. 